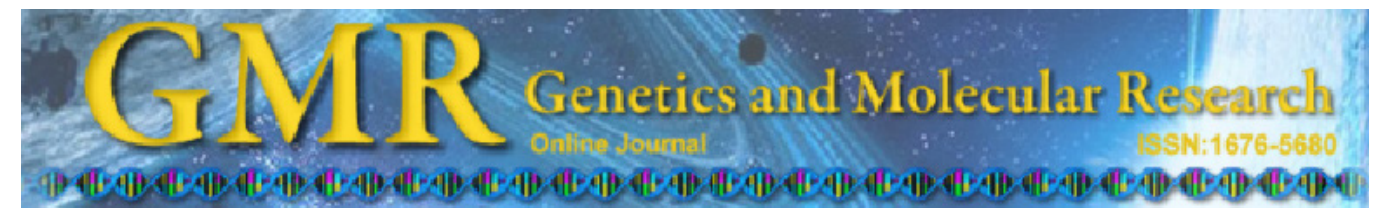

\title{
Comparative genome-wide gene expression analysis of rheumatoid arthritis and osteoarthritis
}

C.Q. Yi ${ }^{1}$, C.H. $\mathrm{Ma}^{1}$, Z.P. Xie ${ }^{2}$, Y. Cao ${ }^{1}$, G.Q. Zhang ${ }^{1}$, X.K. Zhou ${ }^{1}$ and Z.Q. Liu $^{3}$

${ }^{1}$ Department of Orthopaedics, Shanghai First People Hospital, Medical School of Shanghai Jiaotong University, Shanghai, China ${ }^{2}$ Department of Orthopaedics, Shanghai Sixth People Hospital, Medical School of Shanghai Jiaotong University, Shanghai, China ${ }^{3}$ Department of Lymphoma and Myeloma, Division of Cancer Medicine, M.D. Anderson Center for Cancer Immunology Research, University of Texas, Houston, TX, USA

Corresponding author: C.Q. Yi

E-mail: chengqingyidr@gmail.com

Genet. Mol. Res. 12 (3): 3136-3145 (2013)

Received June 5, 2012

Accepted October 15, 2012

Published March 11, 2013

DOI http://dx.doi.org/10.4238/2013.March.11.3

ABSTRACT. Both rheumatoid arthritis (RA) and osteoarthritis (OA)
are complex diseases. Studies and treatment of RA and OA have
mainly focused on individual factors. However, there is still no clear
understanding of their causes and adequate treatment alternatives
are still being sought. We applied gene set-enrichment analysis to
microarray datasets of RA and OA to look for regulatory mechanisms.
We found 32 highly significant pathways, including 18 downregulated
and 14 upregulated pathways associated with RA. We also identified
18 highly significant pathways, including 7 downregulated and 11 up-
regulated pathways associated with OA. Several such pathways were
found in both RA and OA, including an upregulated PPAR signaling
pathway and downregulated leukocyte transendothelial migration.
Regulatory mechanisms in RA seem to be more complex than in OA. 
This information could be useful for diagnosis and treatment of these two diseases.

Key words: Rheumatoid arthritis; GSEA; Osteoarthritis; PPAR signaling pathway

\section{INTRODUCTION}

Rheumatoid arthritis (RA) is a chronic, systemic inflammatory disorder that may affect many tissues and organs but principally attacks synovial joints. The process produces an inflammatory response in the synovium, secondary to the hyperplasia of synovial cells, and leads to excessive synovial fluid as well as the development of pannus in the synovium. The pathology of the disease process often leads to the destruction of articular cartilage and ankylosis of the joints (Willkens, 1987). RA can also produce diffuse inflammation in the lungs, pericardium, pleura, and sclera, as well as nodular lesions, which are most common in the subcutaneous tissue. Although the cause of RA is unclear, autoimmunity plays a pivotal role in both its chronicity and progression, and RA is considered a systemic autoimmune disease (Majithia and Geraci, 2007). It has been suggested that RA involves abnormal B cell-T cell interaction, with presentation of antigens by B cells to T cells via HLA-DR-eliciting T-cell help and consequent production of rheumatoid factors and antibodies to citrullinated peptides (Albano et al., 2001). The inflammation is then driven either by B cell or T cell products, stimulating the release of TNF and other cytokines such as IL-1, IL-15, and IL-6 (Hutchinson et al., 2002).

Osteoarthritis (OA), also known as degenerative arthritis or degenerative joint disease, constitutes a group of mechanical abnormalities involving the degradation of joints, including articular cartilage and subchondral bone. Symptoms may include joint pain, tenderness, stiffness, locking, and effusion. A variety of causes - hereditary, developmental, metabolic, and mechanical - may initiate processes leading to loss of cartilage (O'Connor and Brandt, 1993). When bone surfaces are less well protected by cartilage, bone may be exposed and damaged. Because of the decreased movement secondary to pain, regional muscles may develop atrophy, and ligaments may become more lax. Some investigators believe that various sources of mechanical stress on joints cause OA, including misalignment of bones caused by congenital or pathogenic causes, mechanical injury, overweight, loss of strength in muscle-supporting joints, and impairment of peripheral nerves that leads to sudden or uncoordinated movements that overstress joints (Brandt et al., 2009). However, exercise, including running in the absence of injury, has not been found to increase the risk of developing OA (Bosomworth, 2009), nor has knuckle cracking (deWeber et al., 2011). Lifestyle changes (such as weight loss and exercise) and analgesics are mainstay treatments (Flood, 2010). Although many alternative medicines, including vitamins $\mathrm{A}, \mathrm{C}$, and $\mathrm{E}$, ginger, turmeric, omega-3 fatty acids, chondroitin sulfate, and glucosamine, are purporting to decrease the pain associated with OA, there is no evidence supporting the benefit of these alternative medicines (Rosenbaum et al., 2010).

Previous studies and treatments of RA and OA mainly focused on individual factors involved in these diseases. However, the causes of the diseases are unknown, and satisfactory treatments are lacking. In recent years, gene expression profiling has been used to explore the regulatory mechanisms of RA and OA. Therefore, we performed genome-wide studies, focus- 
ing on the pathways involved in RA and OA as well as the co-expression pattern of associated pathways. We employed microarray datasets associated with RA or OA from the publicly available Gene Expression Omnibus (GEO) and applied gene set-enrichment analysis (GSEA) on the datasets. Our goal is to uncover and compare the regulatory mechanisms of RA and OA. Knowledge of these regulatory mechanisms is essential for both the diagnosis and treatment of these two similar diseases.

\section{MATERIAL AND METHODS}

\section{Data collection and pre-processing}

We used microarray dataset GSE1919 from GEO (www.ncbi.nlm.nih.gov/geo/) (Ungethuem et al., 2010). In this genome-wide dataset, the two disease conditions of RA and OA were investigated and the data were compared to those of normal donors (NDs). For the two disease groups, samples derived from 3 individual patients and 2 pools of patients were hybridized to the microarray platform of the Affymetrix Human Genome U95A Array (HG_U95A, containing 12,626 total probe sets). The raw data are available for download. Five ND replicates (ND_1, ND_2, ND_3, ND_4, and ND_5, from GSM34379 to GSM34391), 5 RA replicates (RA_A, RA_B, RA_x, RA_y, and RA_z, from GSM34398 to GSM34402), and 5 OA replicates (OA_A, OA_B, OA_x, OA_y, and OA_z, from GSM34393 to GSM34397) were used. We conducted two studies: a study of OA, comparing OA and ND samples, and a study of RA, comparing RA and ND samples.

We then performed data pre-processing using a local server with an EMS memory of $64 \mathrm{~Gb}$, using the R v.2.10.1 and the Bioconductor v.2.5.0 software. Each Affymetrix dataset was background adjusted and normalized, and the $\log 2$ probe-set intensities were calculated using the robust multichip averaging algorithm in the Affy package (Gautier et al., 2004).

\section{GSEA}

The category package of Bioconductor v.2.6.0 with the R software was used to perform the GSEA of the pathways and the genes included in the two studies (study of OA and RA) using the Kyoto Encyclopedia of Genes and Genomes (KEGG) database (Chiaretti et al., 2004). First, gene sets represented by $<10$ genes were excluded from the analysis. Next, the $t$-statistical mean of the genes in disease samples and NDs was calculated for each pathway. Lastly, we used a permutation test with 1000 iterations to calculate the corrected $\mathrm{P}$ value of each pathway; the significance level cutoff was set at 0.05 to identify the significant pathways related to RA or OA. The annotation of significant genes in each pathway was performed using the biomaRt package, BioMart v.0.8 rc 3 (Gentleman et al., 2004).

Each identified pathway was grouped into different functional classes, following the classification of identified pathways in pathway map br08901 of the BRITE Functional Hierarchies in the KEGG database (www.genome.jp/kegg-bin/get_htext?br08901.keg). In this database, the KEGG pathways are mapped to 8 functional groups, that is, Global map, Metabolism, Genetic information processing, Environmental information processing, Cellular processes, Organismal systems, Human diseases, and Drug development. 


\section{Clustering analysis}

The clustering of groups or genes was performed using the $\mathrm{R}$ program, based on the expression of the identified genes in each significant pathway using the method of hierarchical clustering with Euclidean distance.

\section{RESULTS}

\section{Significant pathways related to $\mathrm{RA}$ and $\mathrm{OA}$}

With a P value cutoff at 0.05 in the GSEA comparing OA to ND or RA to ND, there were 73 significant pathways related to OA, including 30 downregulated and 43 upregulated pathways. Similarly, there were 85 significant pathways related to RA, including 41 downregulated and 44 upregulated pathways.

After hierarchical clustering with Euclidean distance of groups or genes in each identified pathway, we could identify the significant pathways related to RA or OA. The involved samples were thus clustered into 2 groups: OA $v s$ ND and RA $v s$ ND. As a result, 18 significant pathways were identified as associated with OA, including 7 downregulated and 11 upregulated pathways (Table 1). Moreover, 32 significant pathways associated with RA, including 18 downregulated and 14 upregulated pathways (Table 2). Furthermore, we compared the results of OA and RA to identify the significant pathways common to both OA and RA. As a result, there were three downregulated and upregulated genes (Figure 1). Pathways of interest, including PPAR signaling, existed in the group of upregulated pathways. Based on the expression of 64 significant genes $(\mathrm{P}<0.05)$ in the upregulated PPAR signaling pathway in the study of OA, 10 samples clustered to 2 groups, with OA_y, OA_z, OA_A, OA_B, and OA_x in 1 group and ND_4, ND_1, ND_3, ND_5, and ND_2 in the 2nd group. Similarly, based on the expression of 63 significant genes in the study of RA, a total of 10 samples were grouped as OA_A, OA_z, OA_B, OA_x, and OA_y in the OA group and ND_1, ND_3, ND_4, ND_5, and ND_2 in the ND group.

\section{Functional classification}

In the study of OA, 5 functional groups were involved in the truly significant pathways. Five immune-related pathways, including 1 upregulated pathway (RIG-I-like receptor signaling pathway) and 4 downregulated pathways (leukocyte transendothelial migration, Fc gamma R-mediated phagocytosis, natural killer cell mediated cytotoxicity, and chemokine signaling), were classified into Organismal systems; an endocrine-related pathway (PPAR signaling) was also classified into organismal systems. In the metabolism group, 2 downregulated and 3 upregulated pathways were included. The downregulated glycosaminoglycan catabolism is related to glycan biosynthesis and metabolism, and arachidonic acid metabolism is related to lipid metabolism. The upregulated biosynthesis of unsaturated fatty acids is also related to lipid metabolism. The other 2 pathways, metabolism of xenobiotics by cytochrome P450 and drug metabolism by cytochrome P450, were 
related to xenobiotics biodegradation and metabolism. In the group of human diseases, 3 pathways related to cancer were upregulated: prostate cancer, small cell lung cancer, and pathways in cancer and another endocrine and metabolic disease-related pathway of type II diabetes mellitus. One immune disease-related pathway, primary immunodeficiency, was downregulated. In the group of environmental information processing, there was only 1 upregulated pathway, MAPK signaling, associated with signal transduction. In the group of cellular processes, 1 upregulated pathway, endocytosis, was associated with transport and catabolism.

Table 1. Truly significant pathways related to osteoarthritis.

\begin{tabular}{llll}
\hline Pathways & $\begin{array}{c}\text { Corrected } \\
\text { P values }\end{array}$ & KEGG map 1 & KEGG map 2 \\
\hline Downregualted & & & \\
04670: Leukocyte transendothelial migration & $4.12 \mathrm{E}-12$ & Organismal systems & Immune system \\
00590: Arachidonic acid metabolism & $3.63 \mathrm{E}-06$ & Metabolism & Lipid metabolism \\
04666: Fc gamma R-mediated phagocytosis & $1.11 \mathrm{E}-03$ & Organismal systems & Immune system \\
00531: Glycosaminoglycan degradation & $4.00 \mathrm{E}-03$ & Metabolism & Glycan biosynthesis and metabolism \\
05340: Primary immunodeficiency & $8.00 \mathrm{E}-03$ & Human diseases & Immune diseases \\
04650: Natural killer cell-mediated cytotoxicity & $1.00 \mathrm{E}-02$ & Organismal systems & Immune system \\
04062: Chemokine signaling pathway & $1.10 \mathrm{E}-02$ & Organismal systems & Immune system \\
Upregualted & & & \\
00980: Metabolism of xenobiotics by & & & \\
cytochrome P450 & $1.75 \mathrm{E}-05$ & Metabolism & Xenobiotics biodegradation and metabolism \\
00982: Drug metabolism - cytochrome P450 & $1.83 \mathrm{E}-05$ & Metabolism & Xenobiotics biodegradation and metabolism \\
01040: Biosynthesis of unsaturated fatty acids & $6.80 \mathrm{E}-05$ & Metabolism & Lipid metabolism \\
05215: Prostate cancer & $7.26 \mathrm{E}-05$ & Human diseases & Cancers \\
03320: PPAR signaling pathway & $9.30 \mathrm{E}-04$ & Organismal systems & Endocrine system \\
04010: MAPK signaling pathway & $5.00 \mathrm{E}-03$ & Environmental & \\
& & information processing & Signal transduction \\
04930: Type II diabetes mellitus & $8.00 \mathrm{E}-03$ & Human diseases & Endocrine and metabolic diseases \\
05222: Small cell lung cancer & $1.40 \mathrm{E}-02$ & Human diseases & Cancers \\
04144: Endocytosis & $1.80 \mathrm{E}-02$ & Cellular processes & Transport and catabolism \\
05200: Pathways in cancer & $3.80 \mathrm{E}-02$ & Human diseases & Cancers \\
04622: RIG-I-like receptor signaling pathway & $4.80 \mathrm{E}-02$ & Organismal systems & Immune system \\
\hline
\end{tabular}

KEGG map 1 = first functional classification of related pathways in KEGG database; KEGG map 2 = second functional classification of related pathways in KEGG database.

In the study of RA, 6 immune-related, downregulated pathways and 1 endocrine system-related, upregulated pathway grouped into organismal systems. Twelve upregulated and 1 downregulated pathways grouped into Metabolism, including amino acid metabolism, carbohydrate metabolism, and lipid metabolism. Six downregulated pathways were grouped into human diseases, including 5 immune disease pathways and 1 endocrine and metabolic disease pathway. Only the replication and repair pathway, mismatch repair, was grouped into genetic information processing. Three downregulated pathways were grouped into environmental information processing; 2 were signaling molecule- and interaction-related and 1 was signal transduction-related (e.g., VEGF signaling, cell adhesion molecules, and ECM-receptor interaction). One cell motility-related downregulated pathway, that is, regulation of the actin cytoskeleton, and 1 cell communication-related upregulated pathway pertaining to tight junctions were grouped into cellular processes. The details are listed in Tables 1 and 2 . The significantly related pathways common to both RA and OA were only associated with the functions of organismal systems and metabolism (Table 3). 
Gene expression in rheumatoid arthritis and osteoarthritis

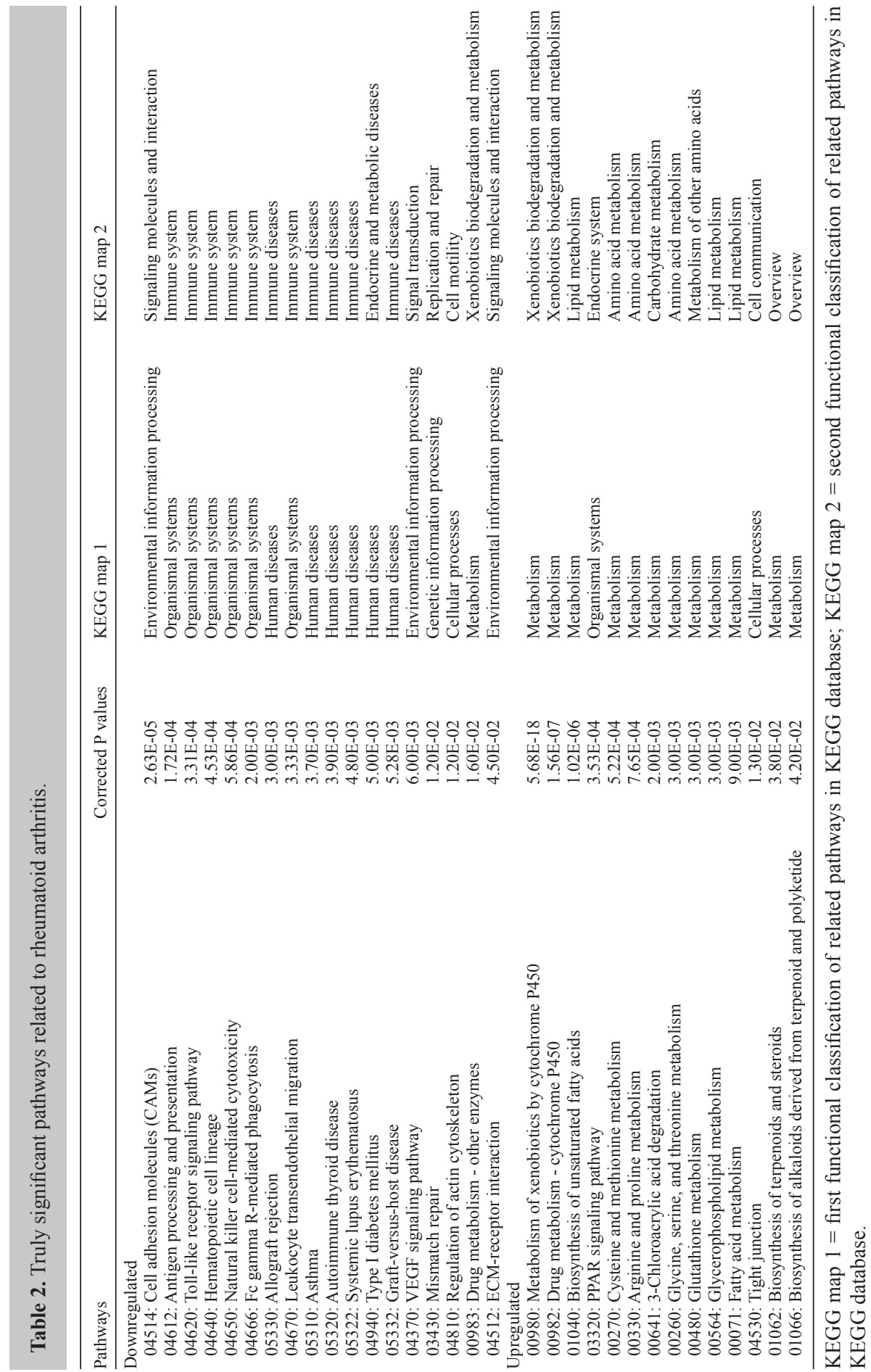




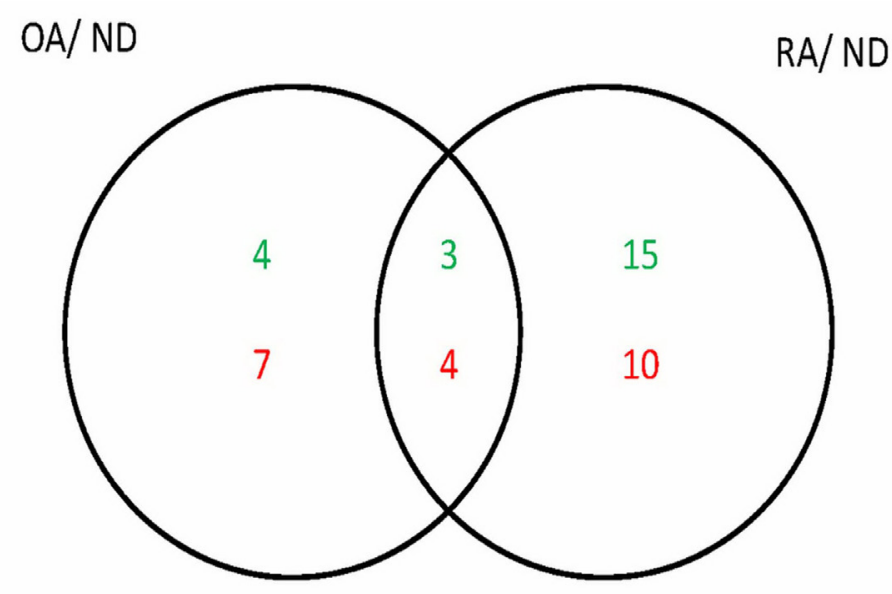

Figure 1. Venn diagram of osteoarthritis (OA) and rheumatoid arthritis (RA) common pathways. OA/ND = comparison of OA samples to normal donors; RA/ND = comparison of RA samples to normal donors. The red color represents the number of upregulated pathways and the green color represents the number of downregulated pathways.

Table 3. Common significantly related pathways to both rheumatoid arthritis (RA) and osteoarthritis (OA).

\begin{tabular}{|c|c|c|c|c|}
\hline Common pathways & $\begin{array}{l}\text { Corrected P values } \\
\text { in OA study }\end{array}$ & $\begin{array}{l}\text { Corrected P values } \\
\text { in RA study }\end{array}$ & KEGG map 1 & KEGG map 2 \\
\hline \multicolumn{5}{|l|}{ Downregulated } \\
\hline 04670: Leukocyte transendothelial migration & 4.12E-12 & $3.33 \mathrm{E}-03$ & Organismal systems & Immune system \\
\hline 04666: Fc gamma R-mediated phagocytosis & $1.11 \mathrm{E}-03$ & $2.00 \mathrm{E}-03$ & Organismal systems & Immune system \\
\hline $\begin{array}{l}\text { 04650: Natural killer cell-mediated } \\
\text { cytotoxicity }\end{array}$ & $1.00 \mathrm{E}-02$ & $5.86 \mathrm{E}-04$ & Organismal systems & Immune system \\
\hline \multicolumn{5}{|l|}{ Upregulated } \\
\hline $\begin{array}{l}\text { 00980: Metabolism of xenobiotics by } \\
\text { cytochrome P450 }\end{array}$ & $1.75 \mathrm{E}-05$ & $5.68 \mathrm{E}-18$ & Metabolism & $\begin{array}{l}\text { Xenobiotics biodegradation } \\
\text { and metabolism }\end{array}$ \\
\hline 00982: Drug metabolism - cytochrome P450 & $1.83 \mathrm{E}-05$ & $1.56 \mathrm{E}-07$ & Metabolism & $\begin{array}{l}\text { Xenobiotics biodegradation } \\
\text { and metabolism }\end{array}$ \\
\hline 01040: Biosynthesis of unsaturated fatty acids & $6.80 \mathrm{E}-05$ & $1.02 \mathrm{E}-06$ & Metabolism & Lipid metabolism \\
\hline 03320: PPAR signaling pathway & $9.30 \mathrm{E}-04$ & $3.53 \mathrm{E}-04$ & Organismal systems & Endocrine system \\
\hline
\end{tabular}

\section{DISCUSSION}

Based on the genome-wide pathway association analysis, the regulatory mechanisms of RA may be more complicated than for OA because there were more pathways involved in the regulation of RA. While rheumatoid arthritis primarily affects joints, problems involving other organs of the body are known to occur. Extra-articular manifestations other than common anemia are clinically evident in approximately $15-25 \%$ of individuals with RA (Turesson et al., 2003). Moreover, people with RA are more prone to atherosclerosis, and the risk of myocardial infarction (heart attack) and stroke is markedly increased (Wolfe et al., 1994; Avina-Zubieta et al., 2008). Because the causes of RA are still unclear, there is no known cure, but many different types of treatments can alleviate symptoms or modify the disease process, for example, pharmacological treatments using disease-modifying antirheumatic drugs, anti- 
inflammatory agents, and analgesics (O'Dell, 2004; Hasler, 2006). In our research, not only the number of associated pathways but also the gene expression patterns of the pathways in the study of RA were different from those in the study of OA. For example, there were 2 distinct gene expression patterns in PPAR signaling. Although the PLTP gene (probe 40081_at) clustered with $U B C$ (probe 1367_f_at or 32334_f_at) both in the study of OA and in the study of RA, several diverse genes clustered in these $\overline{2}$ studies.

There are several common characteristics of RA and OA, including the upregulated PPAR signaling pathway, biosynthesis of unsaturated fatty acids, drug metabolism-related cytochrome P450s, and xenobiotic metabolism by cytochrome P450s as well as the downregulated leukocyte transendothelial migration, Fc gamma R-mediated phagocytosis, and natural killer cell-mediated cytotoxicity (Table 3). Matrix metalloproteinases (MMPs) are thought to be important in destructive joint changes seen in RA and OA, and MMP1 polymorphisms may predict the activity and severity of these diseases (Abd-Allah et al., 2012). In our study, the $M M P 1$ gene was also identified in the significantly upregulated PPAR signaling pathway both in the RA and OA studies. PPARs are members of the nuclear hormone receptor superfamily and have a dominant regulatory role in adipocyte and monocyte differentiation, and PPAR-gamma agonists may provide a new therapeutic approach to treat RA (Kawahito et al., 2000; Ji et al., 2001). RA is a chronic immunological disease in which invasive monocytes and lymphocytes in synovial cells and synovial tissue produce numerous cytokines and inflammatory mediators of paracrine signaling that play a role in the pathological progress in RA patients. The overexpression of IL-18 and IL-18R may be important in the pathogenesis of RA (Shao et al., 2009). Most interleukins, including IL-1R1, IL-1R2, and IL-8, were identified in both RA- and OA-associated pathways, including MAPK signaling and Tolllike receptor signaling. Interconversion of estrone and 17 $\beta$-estradiol and the expression of converting enzymes of the cytochrome P450 family were previously reported to be similar in RA and OA cells (Schmidt et al., 2009). An apoptosis-inducing factor of granzyme B was reported to induce apoptosis of chondrocytes with natural killer cell-like cytotoxicity in RA (Saito et al., 2008). Impairment of neutrophil Fc gamma R cytosolic signaling in active RA could reflect a receptor signaling defect with potential effects on Fc-mediated functions or a defect in calcium ion homeostasis within these cells (Goulding and Guyre, 1992). Leukocyte transendothelial migration and adhesion to RA synovial tissue requires adhesion molecules at the surface of endothelial cells and RA synovial tissue fibroblasts, and the junctional adhesion molecule $\mathrm{C}$ may be crucial for mediating leukocyte recruitment and retention in the RA joint (Rabquer et al., 2008).

In conclusion, both RA and OA are complex diseases. Previous studies and treatments of RA or OA have been mainly focused on the individual factors involved in these diseases; the causes of these diseases remain unclear, and satisfactory treatment options are lacking. Here, we applied GSEA on microarray datasets of RA and OA to uncover the regulatory mechanisms of RA or OA. Finally, 32 significant pathways (including 18 downregulated and 14 upregulated pathways) associated with RA and 18 significant pathways (including 7 downregulated and 11 upregulated pathways) associated with OA. These pathways are involved in diverse biological processes. Moreover, RA and OA had 7 significant pathways in common, including upregulated PPAR signaling and downregulated leukocyte transendothelial migration. Based on the genome-wide pathway association studies, the regulatory mechanisms of RA may be more complicated than for OA. An understanding of the regulatory mechanisms of RA and OA would be essential for the diagnosis and treatment of these 2 diseases. 


\title{
ACKNOWLEDGMENTS
}

\author{
Research supported by the National Natural Science Foundation of China (grant \\ \#30700853).
}

\section{REFERENCES}

Abd-Allah SH, Shalaby SM, Pasha HF, el-Shal AS, et al. (2012). Variation of matrix metalloproteinase 1 and 3 haplotypes and their serum levels in patients with rheumatoid arthritis and osteoarthritis. Genet. Test. Mol. Biomarkers 16: 15-20.

Albano SA, Santana-Sahagun E and Weisman MH (2001). Cigarette smoking and rheumatoid arthritis. Semin. Arthritis Rheum. 31: 146-159.

Avina-Zubieta JA, Choi HK, Sadatsafavi M, Etminan M, et al. (2008). Risk of cardiovascular mortality in patients with rheumatoid arthritis: a meta-analysis of observational studies. Arthritis Rheum. 59: 1690-1697.

Bosomworth NJ (2009). Exercise and knee osteoarthritis: benefit or hazard? Can. Fam. Physician 55: 871-878.

Brandt KD, Dieppe P and Radin E (2009). Etiopathogenesis of osteoarthritis. Med. Clin. North Am. 93: 1-24, xv.

Chiaretti S, Li X, Gentleman R, Vitale A, et al. (2004). Gene expression profile of adult T-cell acute lymphocytic leukemia identifies distinct subsets of patients with different response to therapy and survival. Blood 103: 2771-2778.

deWeber K, Olszewski M and Ortolano R (2011). Knuckle cracking and hand osteoarthritis. J. Am. Board Fam. Med. 24: 169-174.

Flood J (2010). The role of acetaminophen in the treatment of osteoarthritis. Am. J. Manag. Care (16 Suppl Management): S48-S54.

Gautier L, Cope L, Bolstad BM and Irizarry RA (2004). affy - analysis of Affymetrix GeneChip data at the probe level. Bioinformatics 20: 307-315.

Gentleman RC, Carey VJ, Bates DM, Bolstad B, et al. (2004). Bioconductor: open software development for computational biology and bioinformatics. Genome Biol. 5: R80.

Goulding NJ and Guyre PM (1992). Impairment of neutrophil Fc gamma receptor mediated transmembrane signalling in active rheumatoid arthritis. Ann. Rheum. Dis. 51: 594-599.

Hasler P (2006). Biological therapies directed against cells in autoimmune disease. Springer Semin. Immunopathol. 27 : 443-456.

Hutchinson D, O'Leary C, Nixon NB and Mattey DL (2002). Serum complexes of immunoglobulin A-alpha1 proteinase inhibitor in rheumatoid arthritis: association with current cigarette smoking and disease activity. Clin. Exp. Rheumatol. 20: 387-391.

Ji JD, Cheon H, Jun JB, Choi SJ, et al. (2001). Effects of peroxisome proliferator-activated receptor-gamma (PPARgamma) on the expression of inflammatory cytokines and apoptosis induction in rheumatoid synovial fibroblasts and monocytes. J. Autoimmun. 17: 215-221.

Kawahito Y, Kondo M, Tsubouchi Y, Hashiramoto A, et al. (2000). 15-deoxy-delta(12,14)-PGJ(2) induces synoviocyte apoptosis and suppresses adjuvant-induced arthritis in rats. J. Clin. Invest. 106: 189-197.

Majithia V and Geraci SA (2007). Rheumatoid arthritis: diagnosis and management. Am. J. Med. 120: 936-939.

O'Connor BL and Brandt KD (1993). Neurogenic factors in the etiopathogenesis of osteoarthritis. Rheum. Dis. Clin. North Am. 19: 581-605.

O’Dell JR (2004). Therapeutic strategies for rheumatoid arthritis. N. Engl. J. Med. 350: 2591-2602.

Rabquer BJ, Pakozdi A, Michel JE, Gujar BS, et al. (2008). Junctional adhesion molecule C mediates leukocyte adhesion to rheumatoid arthritis synovium. Arthritis Rheum. 58: 3020-3029.

Rosenbaum CC, O'Mathuna DP, Chavez M and Shields K (2010). Antioxidants and antiinflammatory dietary supplements for osteoarthritis and rheumatoid arthritis. Altern. Ther. Health Med. 16: 32-40.

Saito S, Murakoshi K, Kotake S, Kamatani N, et al. (2008). Granzyme B induces apoptosis of chondrocytes with natural killer cell-like cytotoxicity in rheumatoid arthritis. J. Rheumatol. 35: 1932-1943.

Schmidt M, Hartung R, Capellino S, Cutolo M, et al. (2009). Estrone/17beta-estradiol conversion to, and tumor necrosis factor inhibition by, estrogen metabolites in synovial cells of patients with rheumatoid arthritis and patients with osteoarthritis. Arthritis Rheum. 60: 2913-2922.

Shao XT, Feng L, Gu LJ, Wu LJ, et al. (2009). Expression of interleukin-18, IL-18BP, and IL-18R in serum, synovial fluid, and synovial tissue in patients with rheumatoid arthritis. Clin. Exp. Med. 9: 215-221.

Turesson C, O'Fallon WM, Crowson CS, Gabriel SE, et al. (2003). Extra-articular disease manifestations in rheumatoid arthritis: incidence trends and risk factors over 46 years. Ann. Rheum. Dis. 62: 722-727. 
Ungethuem U, Haeupl T, Witt H, Koczan D, et al. (2010). Molecular signatures and new candidates to target the pathogenesis of rheumatoid arthritis. Physiol. Genomics 42A: 267-282.

Willkens RF (1987). Rheumatoid arthritis: clinical considerations in diagnosis and management. Am. J. Med. 83: 31-35.

Wolfe F, Mitchell DM, Sibley JT, Fries JF, et al. (1994). The mortality of rheumatoid arthritis. Arthritis Rheum. 37: 481-494. 